\title{
Anesthesia management in geriatric patients which were interventional bronchoscopy due to central airway obstruction; retrospective analysis
}

\author{
๑Musa Zengin, $₫$ Ramazan Baldemir \\ Health Sciences University Ankara Atatürk Chest Diseases and Thoracic Surgery Training and Research Hospital, Anesthesiology and \\ Reanimation Clinic, Ankara, Turkey
}

Cite this article as: Zengin M, Baldemir R. Anesthesia management in geriatric patients which were interventional bronchoscopy due to central airway obstruction; retrospective analysis. Anatolian Curr Med J 2022; 4(1); 39-43.

\begin{abstract}
Aim: In the endobronchial treatment of tumors that cause obstruction in the tracheobronchial system; Applications such as cryo-recanalization and argon plasma coagulation (APC) are widely used. Patients presenting with central airway obstruction $(\mathrm{CAO})$ require urgent intervention, sharing the airway with the bronchoscopist, severe shortness of breath in patients, and the presence of comorbid diseases make it difficult for anesthetists. This situation becomes more complicated due to pharmacokinetic and pharmacodynamic changes in geriatric patients. In this article, it is aimed to present the anesthesia method in geriatric patients who underwent interventional bronchoscopy due to central airway obstruction.

Material and Method: The files of geriatric patients who underwent interventional procedures for central airway obstruction between January 2021 and September 2021 were reviewed retrospectively. The treatments applied to the patients and the applied anesthetic protocols were recorded.

Results: Forty-five geriatric patients who underwent interventional procedures for CAO were identified. $74 \%$ of the patients were male. 95.6\% of the patients were in the American Society of Anesthesiologists (ASA) III or ASA IV risk group, which we can refer to as the high risk group. It was observed that rapid and short-acting propofol and remifentanil were used in induction and maintenance of anesthesia, rocuronium was used as muscle relaxant, and sugammadex was used to eliminate the residual effect of the muscle relaxant.

Conclusion: Airway management is very complex in severe life-threatening airway stenosis. This situation requires a more comprehensive preoperative evaluation, selection of appropriate short-acting anesthetics, and effective anesthetic monitoring, especially in geriatric patients. In addition, the anesthetist and bronchoscopist should be prepared and in constant communication against complications that may develop.
\end{abstract}

Keywords: Airway obstruction, anesthesia, geriatrics, interventional pulmonology, rigid bronchoscopy

\section{INTRODUCTION}

Rigid bronchoscopy, cryorecanalization and argon plasma coagulation (APC) applications are increasingly used to remove or reduce tumors that cause obstruction in the endobronchial system (1). Dilatation, stent placement, laser and electrocautery are other methods used to reduce or eliminate obstruction (2). General anesthesia is usually required during rigid bronchoscopy (3). However, patients with central airway obstruction $(\mathrm{CAO})$ require urgent intervention, sharing the airway with the bronchoscopist, severe shortness of breath in patients and the presence of comorbid diseases complicate the work of anesthesiologists.
In addition, the fact that the patients are in the geriatric age group makes the work even more complicated and difficult. Geriatric patients are known to be more sensitive to anesthetic agents. It is possible to achieve the desired effect with fewer drugs in geriatric patients, and the drug effect is generally prolonged in the elderly population (4). This difference in geriatric patients is mainly due to the difference in kidney and liver metabolism and, accordingly, changes in pharmacokinetic and pharmacodynamic responses (5-7). Therefore, anesthesia management is very important in geriatric patients presenting with CAO. In this study, it was aimed to present our anesthesia management experience in the treatment of severe airway stenosis in geriatric patients. 


\section{MATERIAL AND METHOD}

The study was initiated with the approval of the Ankara Keçiören Training and Research Hospital Ethics Committee (Date: 28.09.2021, Decision No: 2012-KAEK$15 / 2357$ ), our study was conducted in the $3^{\text {rd }}$ level chest diseases and chest surgery center (Ankara Atatürk Chest Diseases and Thoracic Surgery Training and Research Hospital). All procedures were performed adhered to the ethical rules and principles of the Helsinki Declaration. The files of patients who underwent interventional procedures due to $\mathrm{CAO}$ reviewed retrospectively between January 2021 and September 2021.

Patients aged 65 and over, with ASA score of I-IV and with central airway obstruction were included in the study.

Patients under 65 years of age, patients with ASA score of $\mathrm{V}$ and above, and without central airway obstruction were excluded from the study.

Diagnosis, age, body mass index, gender, history of smoking and alcohol use, ASA score, pre-procedural dyspnea, presence of hemoptysis, mallampati score, concomitant diseases, lesion localizations and treatments, applied anesthetic protocols were recorded.

Data were analyzed using IBM SPSS 25.0 (Armonk, NY: IBM Corp.) statistical package software. Frequency, percentage and standard deviation were used for descriptive statistics

\section{Anesthesia Protocol Applied in CAO}

The patients were evaluated in the anesthesia outpatient clinic at least one day before the interventional procedure. After the ASA physical scores were determined, the patients who signed the written informed consent form underwent an interventional procedure.

Invasive pressure monitoring was also provided by monitoring the patients after routine preparations and performing radial artery cannulation. A $37 \mathrm{Fr}$ and $39 \mathrm{Fr}$ double lumen endobronchial intubation tube was kept ready in case of endobronchial bleeding. After 3 minutes of preoxygenation with $100 \%$ oxygen, $1 \mathrm{mg} / \mathrm{kg} 2 \%$ lidocaine, $50 \mathrm{mg}$ ranitidine, $1 \mathrm{mg} / \mathrm{kg}$ methylprednisolone and 2 $\mathrm{mg} / \mathrm{kg}$ propofol were administered intravenously (iv). Patients were given $40 \mathrm{mcg}$ remifentanil and $0.9 \mathrm{mg} / \mathrm{kg}$ rocuronium iv. After the rigid bronchoscope was inserted into the trachea of the patients by the bronchoscopist, it was connected to the anesthesia circuit and ventilated with balloon ventilation. Anesthesia maintenance was adjusted as propofol $4-6 \mathrm{mg} / \mathrm{kg} /$ hour and remifentanil 2-3 $\mathrm{mcg} / \mathrm{kg} / \mathrm{min}$ as IV infusion. During the procedure, the hemodynamic parameters of the patients were monitored continuously and their arterial blood gases $(\mathrm{ABG})$ values were followed every 10 minutes.
After the procedure was completed, $4 \mathrm{mg} / \mathrm{kg}$ sugammadex was given iv and the rigid bronchoscope was removed. After recovery, the patients were taken to the intensive care unit. Patients who were followed up with nasal O2 and had no complications and had an $\mathrm{SpO} 2$ value above $90 \%$ in room air were sent to the service 2 hours after the procedure.

\section{RESULTS}

Between January 2021 and September 2021, a total of 111 patients underwent interventional procedures for CAO. 47 of them were in the geriatric age group. 2 patients were excluded from the study due to missing data (Figure).

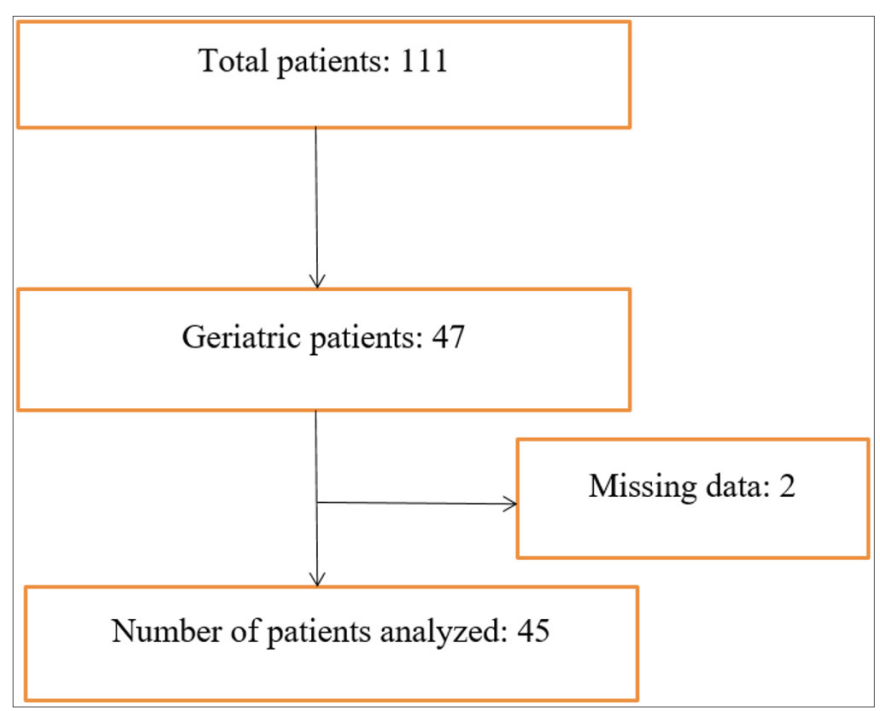

Figure. Flow chart of the patients

Demographic characteristics of patients in the geriatric age group are given in Table 1. 74\% of the patients were male. $95.6 \%$ of the patients were in the ASA III or ASA IV risk group, which we can refer to as the high risk group. In one patient, an interventional procedure was performed under emergency conditions. While $62.2 \%$ of the patients had a history of smoking, $93.4 \%$ did not have a history of alcohol use (Table 1).

Table 2 shows whether the patients had dyspnea and hemoptysis before the procedure, whether they needed nasal oxygen or ventilator, their tracheostomy status and mallampati scores. While $44.4 \%$ of the patients were dependent on nasal oxygen, only $2.2 \%$ were using a home ventilator. Mallampati score was 2 or 3 in $91.1 \%$ of the patients (Table 2).

Additional diseases of the patients are listed in Table 3. Table 4 shows the localization of the pathology causing $\mathrm{CAO}$, the diagnosis of the patients and the procedures for the patients. 


\begin{tabular}{|lc|}
\hline \multicolumn{2}{|c|}{ Table 1. Demographic characteristics of the patients } \\
\hline \multicolumn{1}{|c|}{$\mathbf{n}(\%)$} \\
\hline Gender & $12(26 \%)$ \\
Female & $33(74 \%)$ \\
Male & $71.2 \pm 5.29$ \\
Age ( \pm SD) & $25.81 \pm 4.67$ \\
BMI ( \pm SD) & \\
ASA & $2(4.4 \%)$ \\
II & $26(57.8 \%)$ \\
III & $16(35.6 \%)$ \\
IV & $1(2.2 \%)$ \\
IV-E & $11(24.4 \%)$ \\
Smoking & $17(37.8 \%)$ \\
Yes & $17(37.8 \%)$ \\
No & \\
left & $2(4.4 \%)$ \\
Alcohol use & $42(93.4 \%)$ \\
Yes & $1(2.2 \%)$ \\
\hline No & \\
Left & \\
\hline SD: Standart deviation; BMI: Body mass index; ASA; Amerikan Anestezistler Birliği; \\
E: Emergency
\end{tabular}

\section{Table 3. Additional Diseases of the Patients}

\begin{tabular}{|lc|}
\hline & $\mathbf{n}(\%)$ \\
\hline Lung cancer & $15(33.3 \%)$ \\
Chronic obstructive pulmonary disease & $14(31.1 \%)$ \\
Pyst Covid-19 Infection & $6(13.3 \%)$ \\
Coronary Artery Disease & $17(37.8 \%)$ \\
Arrhythmia & $14(31.1 \%)$ \\
Diabetes Mellitus & $2(4.4 \%)$ \\
Hypothyroidism & $10(22.2 \%)$ \\
Previous Cerebrovascular Event & $1(2.2 \%)$ \\
Chronic renal failure & $3(6.7 \%)$ \\
Extrapulmonary Cancer & $1(2.2 \%)$ \\
\hline
\end{tabular}

\section{DISCUSSION}

In geriatric patients who will undergo rigid bronchoscopy due to severe CAO, general anesthesia is often required to prevent the patient from moving. For this purpose, rapid and short-acting propofol and remifentanil are appropriate choices for induction and maintenance of anesthesia, considering the physiological, pharmacokinetic and pharmacodynamic changes in geriatric patients. It may be preferable to use fast-acting rocuronium to accelerate the passage of the bronchoscope through the vocal cords. We think that sugammadex will be a good choice for rapid recovery and elimination of the residual effect of the muscle relaxant.

The fact that both physicians use a common airway and there are air leaks during ventilation requires continuity of communication between the anesthetist and bronchoscopist during the procedure.

\begin{tabular}{|lc|}
\hline \multicolumn{2}{|l|}{ Table 2. Clinical characteristics of the patients } \\
\hline \multicolumn{2}{|c|}{$\mathbf{n}(\%)$} \\
\hline Dyspnea & $33(73.3 \%)$ \\
Nasal oxygen & $20(44.4 \%)$ \\
Hemoptysis & $5(11.1 \%)$ \\
Home ventilator & $1(2.2 \%)$ \\
Tracheostomy & $3(6.7 \%)$ \\
Mallampati & \\
\multicolumn{1}{|l}{ I } & $3(6.7 \%)$ \\
II & $26(57.8 \%)$ \\
III & $15(33.3 \%)$ \\
IV & $1(2.2 \%)$ \\
\hline
\end{tabular}

\section{Table 4. Diagnosis of the patients, localization of pathology,} procedure performed

\begin{tabular}{|lc|}
\hline \multicolumn{1}{|c|}{$\mathbf{n}(\%)$} \\
\hline DIAGNOSIS & $35(77.7 \%)$ \\
EBL & $6(13.3 \%)$ \\
Tracheal Stenosis & $2(4.4 \%)$ \\
\hline Foreign Body & $2(4.4 \%)$ \\
LOCALIZATION & $14(31.1 \%)$ \\
Trachea & $12(26.6 \%)$ \\
Right Main Bronchus & $17(37.7 \%)$ \\
Left Main Bronchus & $2(4.4 \%)$ \\
\hline Bilateral & $3(6.6 \%)$ \\
APPLIED TREATMENT & $1(2.2 \%)$ \\
APC & $24(53.3 \%)$ \\
MTR & $3(6.6 \%)$ \\
APC+MTR & $4(8.8 \%)$ \\
Dilation & $2(4.4 \%)$ \\
Cryoextraction & $2(4.4 \%)$ \\
Cryoextraction + APC & $4(8.8 \%)$ \\
Cryoextraction + APC + MTR & $2(4.4 \%)$ \\
Biopsy & \\
Foreign Body removal & \\
\hline $\begin{array}{l}\text { EBL: Endobrochial lesion; APC: Argon plasma coagulation; MTR: Mechanical tumor } \\
\text { resection }\end{array}$ & \\
\hline
\end{tabular}

Interventional pulmonology has made significant advances in the diagnosis and treatment of pulmonary pathologies in recent years (8). Along with these developments, the anesthesia method to be applied is also important. In the literature, performing rigid bronchoscopy under deep sedation where spontaneous breathing is preserved is reported as an alternative to general anesthesia. However, the possibility of hypoventilation in deep sedation, laryngospasm, insufficient relaxation of the laryngeal muscles, coughing or movement of the patient complicates the insertion of the rigid bronchoscope into the trachea and the work of the team (9). However, geriatric patients presenting with $\mathrm{CAO}$ usually have a high rate of comorbidities. Therefore, the possibility of complications related to anesthesia is high $(10,11)$.

A safe and controlled airway should be established before the interventional procedure. Alternative airway 
equipment such as small-diameter intubation tube, double-lumen intubation tube (DLT) and high-frequency jet ventilator should be ready for acute complications such as bleeding, pneumothorax, and airway obstruction (9). Massive bleeding, especially during the procedure, is a serious life-threatening complication. In this case, DLT, which is frequently applied in our clinic, can be life-saving.

Especially in cases with severe stenosis with limited reserves, hypoxia and cardiopulmonary arrest may develop after the compensation mechanisms are depressed (12). In addition, it should be kept in mind that masses located in the anterior mediastinum may cause tracheal obstruction and sudden cardiovascular collapse during induction due to compression in the supine position (13). For these reasons, the anesthesia method to be preferred should create a rapid and sufficient level of anesthesia, and at the same time, short-acting anesthetic drugs that will provide rapid recovery should be selected (14).

Total intravenous anesthesia (TIVA) should be considered as the first option in the maintenance of anesthesia instead of inhalation anesthesia for reasons such as air leaks in ventilation with a rigid bronchoscope and the need for apnea periods during the procedure (9). Propofol and remifentanil infusion are frequently used together in TIVA due to the short half-life and rapid recovery of patients.

The adequacy of ventilation should be checked with $\mathrm{SpO} 2$ and end-tidal CO2 monitoring. The presence of hypercapnia is a strong indicator for inadequate ventilation (14). However, the presence of air leaks requires the end-tidal $\mathrm{CO} 2$ value to be measured with an accurate technique. Transcutaneous CO2 has been used for hypercapnia monitoring in recent years. However, in cases where this technique cannot be applied, it is very important to monitor acid-base balance, pCO2 and pO2 with $\mathrm{ABG}$ analysis at regular intervals. Invasive arterial monitoring will be useful for close $\mathrm{ABG}$ monitoring and instant hemodynamic evaluation.

Bispectral index monitoring can be used as an appropriate method for the evaluation of depth of anesthesia $(15,16)$. In addition, TOF (train of four) monitoring may be useful in these patients for the evaluation of neuromuscular blockade efficiency (17). It was seen that these techniques were not used in our study, but we think that the use of these two techniques would be beneficial in terms of increasing anesthesia safety.

Reversing the effect of post-procedure muscle relaxants with neostigmine is a frequently used method. Sugammadex, on the other hand, reverses the effect of muscle relaxation faster than neostigmine. In addition, cholinergic side effects are not observed and residual muscle relaxant effects are less common $(18,19)$.

\section{CONCLUSION}

As a result, anesthesia management in airway stenosis becomes complicated because the anesthetist and bronchoscopist use the airway together and the procedure is high-risk. Addition of physiological, pharmacokinetic and pharmacodynamic changes and comorbidities in geriatric patients to this situation can put a strain on the anesthetist. Therefore, a comprehensive preoperative evaluation, selection of appropriate short-acting anesthetics, effective anesthetic monitoring, and good perioperative communication with the operating team are very important in preventing complications in geriatric patients.

\section{ETHICAL DECLARATION}

Ethics Committee Approval: The study was initiated with the approval of the Ankara Keçiören Training and Research Hospital Ethics Committee (Date: 28.09.2021, Decision No: 2012-KAEK-15/2357).

Informed Consent: Because the study was designed retrospectively, no written informed consent form was obtained from patients.

Referee Evaluation Process: Externally peer-reviewed.

Conflict of Interest Statement: The authors have no conflicts of interest to declare.

Financial Disclosure: The authors declared that this study has received no financial support.

Author Contributions: All of the authors declare that they have all participated in the design, execution, and analysis of the paper, and that they have approved the final version.

\section{REFERENCES}

1. de Lima A, Kheir F, Majid A, Pawlowski J. Anesthesia for interventional pulmonology procedures: a review of advanced diagnostic and therapeutic bronchoscopy. Can J Anaesth 2018; 65: 822-36.

2. Okiror L, Jiang L, Oswald N, et al. Bronchoscopic management of patients with symptomatic airway stenosis and prognostic factors for survival. Ann Thorac Surg 2015; 99: 1725-30.

3. Bakan M, Topuz U, Umutoglu T, et al. Remifentanil-based total intravenous anesthesia for pediatric rigid bronchoscopy: comparison of adjuvant propofol and ketamine. Clinics (Sao Paulo) 2014; 69: 372-7.

4. Kanonidou Z, Karystianou G. Anesthesia for the elderly. Hippokratia 2007; 4: 175-7.

5. Cavalieri TA. Managing pain in geriatric patients. J Osteopathic Med 2007; 107: E10-E16.

6. Gloth FM 3rd. Pain management in older adults: prevention and treatment. J Am Geriatr Soc 2001; 49: 188-99.

7. Cavalieri TA. Pain management in the elderly. J Am Osteopath Assoc 2002; 102: 481-5.

8. Arias S, Lee HJ. The future of interventional pulmonology. Semin Respir Crit Care Med 2014; 35: 6. 
9. Dincq AS, Gourdin M, Collard E, et al. Anesthesia for adult rigid bronchoscopy. Acta Anaesthesiol Belg 2014; 65: 95-103.

10. Chadha M, Kulshrestha M, Biyani A. Anaesthesia for bronchoscopy. Indian J Anaesth 2015; 59: 565-73.

11. Putz L, Mayné A, Dincq AS. Jet Ventilation during Rigid Bronchoscopy in Adults: A Focused Review. Biomed Res Int. 2016; 2016: 4234861

12. Ramaswamy AH, Kurdi MS, Shaikh SI, Deva RS, Raza SU. Awake rigid bronchoscopy for post intubation tracheal stenosis. Anaesth Pain \& Intensive Care 2014; 18: 299-301.

13. Bolliger CT, Mathur PN, Beamis JF, et al. ERS/ATS statement on interventionalpulmonology. European Respiratory Society/ American Thoracic Society. Eur Respir J 2002; 19: 356-73.

14. Salman Önemli C, Çatal DA. Trakeal Stenozun Rijit Bronkoskop ve Balon Dilatasyonla Tedavisinde Total İntravenöz Anestezi Kullanımı: Dört Olgunun Sunumu. GKDA Derg 2015; 21, 4: 174-9.

15. Avidan MS, Zhang L, Burnside BA, et al. Anesthesia awareness and the bispectral index. N Engl J Med 2008; 358: 1097-108.

16. Zorrilla-Vaca A, Healy RJ, Wu CL, Grant MC. Relation between bispectral index measurements of anesthetic depth and postoperative mortality: a meta-analysis of observational studies. Can J Anaesth 2017; 64: 597-607.

17. Arıkan M, Ulus F, Sazak H, Şavkıoğlu E, Pehlivanoğlu P, Cengiz Z. Yaşlilığın rokuronyum ile sağlanan nöromüsküler bloğa etkisinin train of four (TOF) ve posttetanik count (PTC) ile değerlendirilmesi. Ortadoğu Tip Dergisi 2011; 3: 61-5.

18. Honing G, Martini CH, Bom A, et al. Safety of sugammadex for reversal of neuromuscular block. Expert Opin Drug Saf 2019; 18: 883-91.

19. Brueckmann B, Sasaki N, Grobara P, et al. Effects of sugammadex on incidence of postoperative residual neuromuscular blockade: a randomized, controlled study. Br J Anaesth 2015; 115: 743-51. 